\title{
Eficacia y seguridad de las aminoquinolinas para el tratamiento de infecciones a SARS-CoV-2: Una revisión sistemática de la literatura
}

\author{
Efficacy and safety of aminoquinolines for treating patients infected with \\ SARS-COV-2: A systematic review of the literature \\ Cristian Pavez N. MD. ${ }^{1, *}$, Felipe Martínez L. MD. MSc. ${ }^{1,2}$, Dominique Boisier R. MD. ${ }^{1}$, Jaime Vidal M. MD. ${ }^{1}$, \\ Eduardo Labarca M. MD Mg1 \\ Unidad de Cuidados Intensivos Generales, Hospital Naval Almirante Nef. Viña del Mar, Chile. \\ 2 Escuela de Medicina, Facultad de Medicina, Universidad Andrés Bello. Viña del Mar, Chile.
}

Conflictos de Interés: Los autores declaran no tener ningún conflicto de interés que declarar.

Financiamiento: Este estudio fue realizado en forma independiente, sin recibirse fondos para su ejecución.

Fecha de recepción: 25 de septiembre de2021 / Fecha de aceptación: 12 de octubre de 2021

\begin{abstract}
Introduction: Amoniquinolines such as hydroxycholorquine have shown to inhibit SARS-CoV2 replication in-vitro. However their benefit in clinical terms are controversial. Objectives: To synthesize the available evidence regarding the use of aminoquinolines for treating patients with COVID-19. Methods: A systematic review was undertaken. Five databases were searched for randomized trials assessing chloroquine o hydroxychloroquine in the treatment of patients infected with SARS-CoV-2. All studies were assessed using the criteria endorsed by the Cochrane Collaboration. Metaanalyses were undertaken whenever possible. Results: The literature search yielded 852 references, and 5 randomized trials were included. All were at high risk of bias. All studies included several cointerventions, such as antibiotics, steroids and antivirals and only two of them included critically-ill patients. When meta-analyses were conducted, aminoquinolines showed no benefit in terms of viral clearance or clinical improvement. However, a significant increase in the incidence of adverse events was observed (RR 3.11, 95Cl\% 1.64-5.89, p<0.001). Discussion: The inhibitory properties of aminoquinolines do not seem to translate in clinical benefits amongst patients with COVID-19. The perceived high risk of bias of included studies along the significant increase in terms of adverse events recommends against the routine use of these drugs for treating patients with COVID-19.
\end{abstract}

Key words: Aminoquinolines, chloroquine, hydroxychloroquine, COVID-19, SARS-CoV-2, Treatment.

\section{RESUMEN}

Introducción: Las aminoquinolinas como hidroxicloroquina han mostrado eficacia en inhibir la replicación del SARS-CoV-2 in vitro. Sus efectos en pacientes reales son controversiales. Objetivos: Sintetizar la evidencia disponible respecto al uso de aminoquinolinas en el tratamiento de pacientes con COVID-19. Metodología: Revisión sistemática de la literatura de ensayos clínicos aleatorizados (ECA) que evaluaron la eficacia de cloroquina o hidroxicloroquina en el tratamiento de pacientes infectados con SARS-CoV-2. Se buscó evidencia en 5 bases de datos, sólo incluyendo ECAs que fueron evaluados mediante los criterios de calidad de la colaboración Cochrane. Los resultados fueron sintetizados en un metaanálisis siempre que fue posible. Resultados: Se evaluaron 852 referencias, incluyéndose 5 ensayos clínicos aleatorizados con alto riesgo de sesgo. Sólo dos estudios incluyeron pacientes críticamente enfermos. No se apreciaron beneficios en términos de aclaramiento viral o mejoría clínica al realizar el metaanálisis. Se detectó un incremento significativo en la incidencia de reacciones adversas (RR 3,11, IC95\% 1,64-5,89, p < 0,001), si bien la mayoría fueron reportadas como leves. Discusión: Las aminoquinolinas poseen efectos inhibitorios in-vitro que no han demostrado traducirse en beneficios clínicos. El incremento significativo en la incidencia de reacciones adversas asociado a la baja calidad de los estudios evaluados no permite recomendar este tratamiento para pacientes con COVID-19.

Palabras clave: Aminoquinolinas, cloroquina, hidroxicloroquina, COVID-19, SARS-CoV-2, tratamiento. 


\section{Introducción}

E n diciembre de 2019, varios centros de salud de Wuhan, China, reportaron la aparición de casos de neumonía compatibles con SARS[1],[2]. Durante los primeros días de enero de 2020 se aisló una nueva cepa de coronavirus, el coronavirus del síndrome respiratorio agudo severo (SARS-CoV-2) y confirmó la existencia de un nuevo síndrome clínico similar al SARS que fue acuñada COVID-19. Si bien el curso clínico de la enfermedad fue descrito como benigno en la mayoría de los casos, individuos mayores (> 60 años) y personas con enfermedades médicas preexistentes mostraron peor pronóstico y desenlaces en salud[3],[4]. A la fecha, cientos de miles de personas han adquirido la enfermedad en el mundo y, si bien la mayoría se presenta como un resfrío común, una proporción de pacientes desarrolla una enfermedad mayor que amerita la hospitalización (10\% a 15\%) y hasta 5\% requiere ventilación mecánica invasiva por el desarrollo de falla respiratoria. La letalidad promedio de la afección es de 3,5\%, pero es desproporcionadamente alta entre pacientes con factores de riesgo (edad avanzada, con comorbilidades, elevación de dímero D o troponina ultrasensible al ingreso) y entre aquellos que requieren ventilación mecánica invasiva[5]-[8].

Considerando la alta contagiosidad del SARS-CoV-2 y la posibilidad de causar insuficiencia respiratoria con requerimientos de ventilación mecánica con la subsecuente mortalidad asociada, existe un importante interés en la literatura internacional por el uso de tratamientos que permitan evitar estos desenlaces[9]-[11]. Entre estos últimos se ha mencionado a dos integrantes de la familia de las aminoquinolinas como potenciales alternativas terapéuticas para casos de COVID-19[12],[13]. Tanto la cloroquina como la hidroxicloroquina, fármacos antimaláricos con actividad inmunosupresora muy conocida y de amplia utilización en el tratamiento de diversas enfermedades autoinmunes han demostrado actividad supresora del virus en modelos in-vitro[14]-[16]. En células Vero-E6 se ha apreciado que bajas concentraciones de cloroquina fueron capaces de inhibir la replicación viral[17],[18]. El mecanismo de acción estaría basado en la alcalinización de los endosomas, así como alteraciones en la glicosilación de receptores de angiotensina 2 que son cruciales para el ingreso del virus a la célula[14],[19],[20]. En estos modelos también se ha descrito un aumento en la actividad de linfocitos $T$ regulatorios, importantes en la modulación de la respuesta inmune posterior[14],[21]. Similares efectos se han descrito para hidroxicloroquina, más con menor potencial de toxicidad y requerimientos de dosis para llegar a similares resultados. No obstante, existe significativa incertidumbre en términos de si los efectos de estos fármacos se traducen en un beneficio clínico para los pacientes, especialmente considerando que algunos estudios no han demostrado beneficio entre pacientes con COVID-19[22],[23]. Por este motivo, se hace necesario realizar una síntesis de la evidencia disponible para evaluar la eficacia y seguridad de cloroquina e hidroxicloroquina, ya sea en monoterapia o en asociación con otros fármacos, en el tratamiento de infección a COVID-19.

\section{Metodología}

\section{Estrategia de búsqueda}

Con el fin de sintetizar la evidencia disponible respecto a la eficacia de cloroquina o hidroxicloroquina en el tratamiento de infección moderada a severa por SARS-CoV-2, se realizó una revisión sistemática de la literatura en diversas bases de datos. Se realizó una búsqueda iterativa en los repositorios PubMED/ MEDLINE, EMBASE, Epistemonikos, LILACS y Cochrane CENTRAL desde su creación hasta mayo de 2020. Sólo se consideraron estudios primarios con diseño de ensayos clínicos aleatorizados o ensayos clínicos controlados para informar la práctica clínica. Se buscó además literatura no-publicada mediante la revisión de repositorios de protocolos de ensayos clínicos (como ClinicalTrials.gov) y la base de datos de la iniciativa OpenGrey. La presente revisión sistemática fue efectuada siguiendo las pautas PRISMA para el reporte de revisiones sistemáticas de la literatura[24].

Para estos fines se integraron los términos Coronavirus, Coronavirus Infections, COVID-19, Severe Acute Respiratory Syndrome Coronavirus 2, SARS-COV-2, Wuhan coronavirus, Hydroxychloroquine, Chloroquine, Placebo, Usual Care, Mechanical Ventilation, Orotracheal Intubation, Intubation, Death Mortality, Survival, Hospital Stay, Radiological Progression, Virological Cure. Se muestra una síntesis de los términos de búsqueda utilizados en la Tabla 1 en el Anexo. No se aplicaron restricciones de idioma para la inclusión de estos estudios. Todos los estudios pasaron por varios filtros para su inclusión. En primer lugar, se evaluó la pertinencia del estudio a partir del análisis de los títulos, para luego pasar a un segundo filtro de evaluación de aspectos metodológicos esenciales y de adecuación poblacional basado en el estudio del resumen. Los textos potencialmente relevantes fueron seleccionados a partir de este procedimiento para evaluación del texto completo.

También se consideró listados de referencias de repositorios de evidencia secundaria, incluyendo revisiones sistemáticas de la literatura conducidas entre pacientes adultos con infección confirmada a SARS-CoV2 que requirieran hospitalización. Asimismo se consideró a guías clínicas y recomendaciones de sociedades científicas internacionales, incluyendo a la Sociedad Chilena de Medicina Intensiva (SOCHIMI), Sociedad Chilena de Infectología (SOCHINF), Sociedad Chilena de Enfermedades Respiratorias (SER-Chile), Organización Mundial de la Salud (WHO), European Society of Intensive Care Medicine (ESICM), Society of Critical Care Medicine y la Intensive Care Society. Asimismo se evaluó la bibliografía de los estudios primarios incluidos para la detección de referencias adicionales.

\section{Evaluación de calidad de estudios}

Después del tamizaje inicial y la consideración de los criterios de inclusión antes mencionados, todos los ensayos fueron evaluados por los revisores, usando los criterios propuestos por la Colaboración Cochrane para evaluar la calidad de estudios orientados a intervenciones[25]. En resumen, estos criterios incluyen la ocultación de la secuencia de asignación generada, el nivel de enmascaramiento usado en cada ensayo, el número de pacientes perdidos durante el seguimiento y el análisis realizado bajo el principio de intención de tratar. Cada estudio fue evaluado de forma independiente y sus hallazgos fueron discutidos después, con el fin de proporcionar un resumen para clasificar cada estudio de acuerdo a su probabilidad de sesgo. Se consideraron tres categorías respecto al riesgo de sesgo en cada dominio: bajo, moderado y alto riesgo de 


\begin{tabular}{|c|c|c|c|}
\hline Paciente & Intervención & Comparación & Desenlace \\
\hline Coronavirus & Hydroxychloroquine & Placebo & Mechanical ventilation \\
\hline Coronavirus infections & Chloroquine & Usual Care & Orotracheal intubation \\
\hline Severe acute respiratory syndrome coronavirus 2 & & & Intubation \\
\hline SARS-COV-2 & & & Mortality \\
\hline COVID-19 & & & Death \\
\hline \multirow[t]{6}{*}{ Wuhan coronavirus } & & & Survival \\
\hline & & & Radiological progression \\
\hline & & & Virological cure \\
\hline & & & Viral load \\
\hline & & & Hospital stay \\
\hline & & & Respiratory failure \\
\hline
\end{tabular}

sesgo, los que después fueron sintetizados en un gráfico de riesgo de sesgo.

\section{Extracción de datos}

Se aplicó un formulario de extracción de datos para todos los estudios incluidos. Brevemente, este formulario incluyó la siguiente información de aspectos metodológicos y resultados de los estudios incluidos:

a) Características de los pacientes

- Número de participantes (total y por grupo).

- Promedio de edad de los participantes y desviación estándar.

- Ambiente de desarrollo del estudio (ambulatorio, hospitalización en sala, hospitalización en UCI).

- Relación Pa/Fi promedio de los pacientes incluidos acompañado de su desviación estándar.

- Comorbilidades relevantes (hipertensión esencial, insuficiencia renal crónica, insuficiencia cardiaca, enfermedad pulmonar obstructiva crónica, diabetes mellitus) (n, \%).

b) Resultados de los estudios

- Proporción de los pacientes que fallecieron durante la hospitalización (n, \%).

- Proporción de pacientes que presentaron deterioro clínico o requirieron ventilación mecánica $(n, \%)$.

- Proporción de pacientes que presenta progresión radiológica durante su seguimiento ( $n, \%)$.

- Proporción de pacientes que negativizan hisopado para SARS-CoV-2.

- Promedio de la duración de la ventilación mecánica (días), desviación estándar y rangos.

- Promedio de la duración de estadía hospitalaria (días), desviación estándar y rangos.

- Proporción de pacientes que presentan arritmias, incluyendo prolongación de intervalo QT (n, \%).

c) Evaluación de la calidad

- Ocultamiento de la asignación aleatoria (Si/No).

- Nivel de enmascaramiento (participantes, evaluadores, esta- dísticos, auspiciadores).

- Proporción de pacientes perdidos en el seguimiento (n, \%).

- Análisis realizado bajo el principio de intención de tratar (Sí) No).

- Otras fuentes de sesgo (comentario abierto).

\section{Análisis de datos}

Siempre que fue apropiado, los datos fueron sintetizados en un metaanálisis. Para resultados binarios, se calculó una estimación estándar del riesgo relativo o la razón de disparidad (odds ratio), acompañado de sus intervalos de confianza correspondientes al 95\%. Para resultados continuos, como la duración total de la ventilación mecánica o la estadía hospitalaria, se empleó la diferencia de medias ponderadas como un resumen estadístico con su intervalo de confianza correspondiente también al 95\%.

La heterogeneidad fue evaluada mediante el uso de estadísticos Q e 12 de Cochrane. El estadísico 12 proporciona una medición de la variación entre los estudios que no se puede atribuir a la casualidad y se expresa como porcentaje. A menudo se ha categorizado como sigue: < 25\% bajo, 25 a $50 \%$ moderado y $>50 \%$ alto[25]-[27]. Se empleó un modelo de efectos fijos para permitir agrupar los resultados en casos de baja heterogeneidad de resultados, mientras que un modelo de efectos aleatorios fue preferido de encontrarse alta heterogeneidad entre ensayos. Se programaron análisis de subgrupo para explorar posibles fuentes de heterogeneidad, incluyendo el uso de hidroxicloroquina o cloroquina de forma exclusiva o en asociación a otra intervención (como azitromicina), el tipo de estudio evaluado (ensayo clínico aleatorizado versus otro).

El sesgo de publicación fue evaluado mediante un gráfico en embudo (funnel plot). Todos los análisis fueron realizados mediante Review Manager (RevMan) [programa computacional], versión 5.2, Copenhagen: The Nordic Cochrane Centre, The Cochrane Collaboration, 2012.

\section{Resultados}

Se revisaron 852 registros de estudios potencialmente re- 
levantes para la revisión sistemática, de los cuales 315 correspondieron a entradas independientes. En base a los criterios de selección antes propuestos, se revisaron los resúmenes de 88 publicaciones originales, quedando finalmente 21 seleccionadas para la evaluación de texto completo. De éstas, 16 fueron excluidas por los siguientes motivos: 5 por corresponder a estudios in vitro, 6 por corresponder a revisiones panorámicas de la literatura, 4 por corresponder a estudios observacionales y una por corresponder a una carta al editor finalmente resultando en 5 publicaciones para informar la práctica clínica y realizar un metaanálisis[13],[28]-[32]. De estas publicaciones, 4 se encontraban escritas en inglés y una en chino, por lo que fue traducida para obtener la información necesaria para el análisis de calidad. Se muestra un detalle del proceso de selección de los estudios en la Figura 1.

\section{Características de los estudios seleccionados}

Cuatro de los artículos seleccionados correspondieron a ensayos clínicos aleatorizados, mientras que el último utilizó un diseño de ensayo clínico controlado asignando intervenciones según centro de consulta y preferencias del paciente. Los estudios variaron en tamaño desde los 36 a 150 participantes. Todos los estudios fueron realizados entre pacientes con enfermedad leve a moderada, excluyéndose de la investigación a pacientes críticos con infección a SARS-CoV-2. Los estudios de Silva[30] y Tang[32] incluyeron pacientes con enfermedad severa COVID-19, si bien el último sólo ingresó dos casos de
COVID-19 severo tras una modificación a posteriori del protocolo.

Cuatro de los cinco estudios evaluaron hidroxicloroquina en distintos esquemas contra terapia usual[13],[28],[29],[32]. Sólo uno de los estudios seleccionados evaluó a cloroquina en esquema de 600 mg cada 12 h por 10 días[30]. Se comparó la eficacia de estos tratamientos contra cuidado usual en cuatro de los cinco estudios, el que incluyó uso de corticoides, antibióticos, antivirales, inmunoglobulinas y oxígeno a discreción de los médicos tratantes. El estudio de Silva y colaboradores utilizó una dosis menor de cloroquina como comparador (cloroquina $450 \mathrm{mg} \mathrm{c/12h}$ el día 0, luego 450 mg/d hasta el cuarto día y placebo los días restantes).

\section{Riesgo de sesgo de los estudios incluidos}

Todos los estudios incluidos en la presente revisión presentaron un alto riesgo de sesgo. Las principales limitaciones tuvieron que ver con la falta de enmascaramiento de tratamientos de tanto participantes como evaluadores, así como falta de ocultamiento de secuencia de aleatorización en tres de los cinco estudios incluidos. Todos los estudios confirmaron la presencia de SARS-CoV-2 mediante reacción en cadena de la polimerasa, más el estudio de Silva[30] realizó una confirmación posterior a la inclusión de estos pacientes en el estudio. Se reportó que la infección fue confirmada en 62 de los pacientes incluidos (76,5\% de los casos).

La mayoría de los estudios incluidos reportó una baja fre-

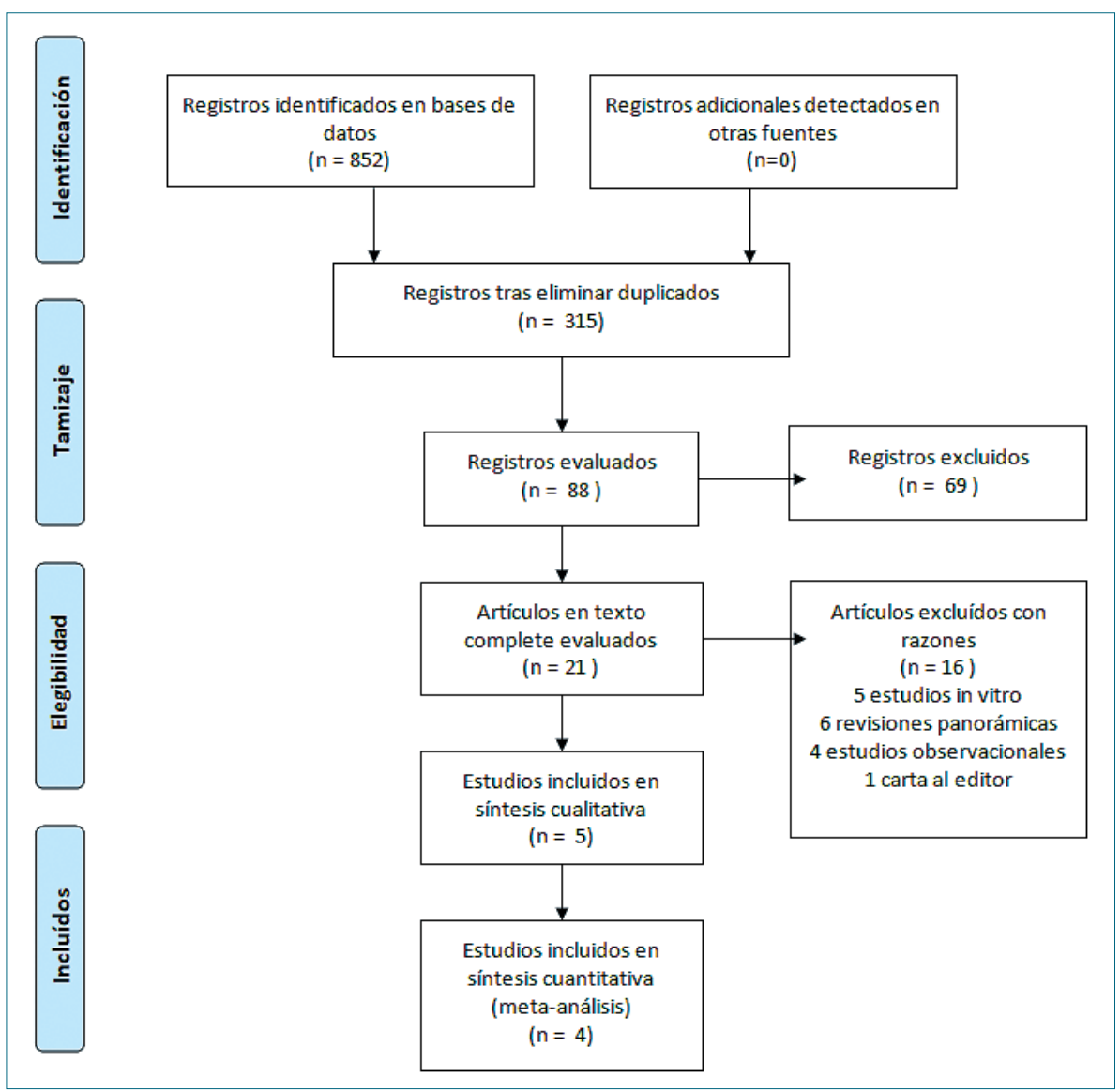

Figura 1. Flujograma de búsqueda. 
cuencia de pérdidas de seguimiento, la que llegó a un máximo de $15 \%$. No obstante, destacó que la mayoría de los pacientes perdidos ocurrieron en el grupo asignado a una aminoquinolina. En dos trabajos no hubo claridad en la proporción de pacientes perdidos del seguimiento. Se muestra un resumen de la valoración de riesgo de sesgo de los estudios incluidos en la Figura 2.

\section{Desenlaces}

\section{Hidroxicloroquina contra terapia usual}

Cuatro de los estudios incluidos condujeron sus intervenciones contra cuidado usual[13],[28],[29],[32]. No obstante, todos incluyeron la aplicación de cointervenciones a discreción del médico tratante, incluyendo antibióticos (ceftriaxona, azitromicina, entre otros), antivirales (oseltamivir, ribavirina, lopinavir/ritonavir, etc), glucocorticoides, inmunoglobulinas y oxigenoterapia. La forma de utilización de hidroxicloroquina fue altamente variable, con dosis desde $200 \mathrm{mg}$ cada $12 \mathrm{~h}$ hasta esquemas de $1.200 \mathrm{mg}$ por una vez y luego $800 \mathrm{mg}$ al día por 2 a 3 semanas.

\section{Negativización del hisopado}

Tres estudios reportaron la realización de hisopados de control entre los pacientes incluidos. Las fechas de control fueron variables, oscilando desde los 6 a los 28 días. El único trabajo que reportó diferencias estadísticamente significativas fue el de Gautret[29] y colaboradores, que reportó que 14 de los 26 pacientes tratados presentaron un hisopado negativo a los 6 días de seguimiento, comparado con 2 de los 16 pacientes asignados al grupo control. No obstante, el metaanálisis de 222 pacientes respecto este desenlace no mostró diferencias estadísticamente significativas en la probabilidad negativizar el hisopado en cualquier momento de control (Riesgo Relativo, RR: 1,08, IC95\% 0,77-1,51, $p=0,66)$. Existió importante heterogeneidad estadística en los resultados de los trabajos, fundamentalmente explicada por el estudio de Gautret[29] (Q de Cochrane $p=0,02,\left.\right|^{2} 73 \%$ ). Se muestra el Forest Plot del metaanálisis en la Figura 3.

\section{Mortalidad}

Sólo un estudio informó cifras de mortalidad comparando hidroxicloroquina contra cuidados usuales. No obstante, no se apreciaron eventos en dicha investigación, por lo que no es posible referirse a este desenlace respecto a hidroxicloroquina.

\section{Progresión de la enfermedad clínica}

Dos estudios[13],[28] reportaron la probabilidad de progre-

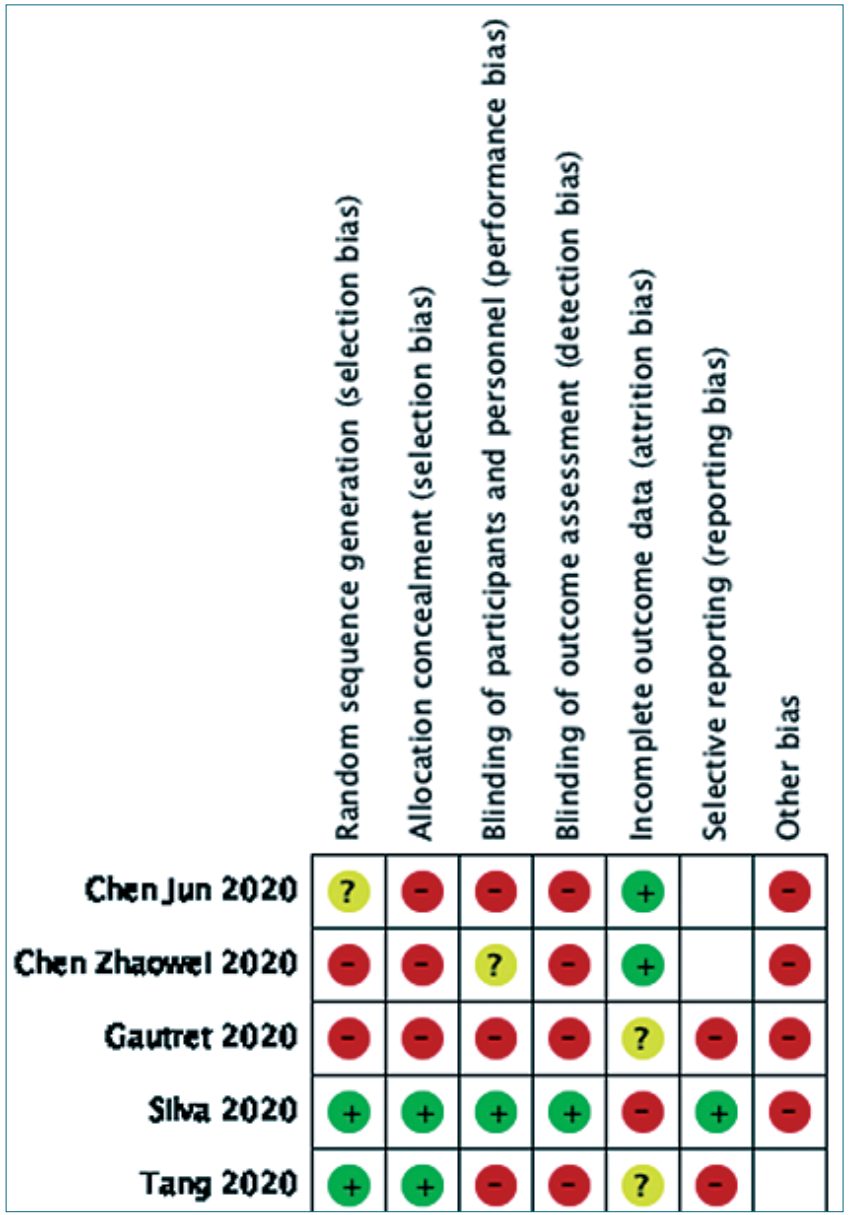

Figura 2. Riesgo de sesgo de estudios evaluados.

sión de la enfermedad, desenlace que incluyó a pacientes que requirieron ingreso a hospitalización en unidades de cuidado intensivo o ventilación mecánica invasiva. Al realizar el metaanálisis de estos estudios que incluyó 92 participantes, no se apreció evidencia de que hidroxicloroquina modificara la probabilidad de progresión de la enfermedad (RR: 0,55, IC95\% 0,02 - 14,21 $p=0,72)$. Al igual que en el metaanálisis anterior se apreció importante heterogeneidad estadística en los resultados observados (Q de Cochrane $p=0,12,1^{2}=58 \%$ ). Se muestra el Forest Plot del metaanálisis en la Figura 4.

\section{Progresión radiológica de la enfermedad}

Dos estudios realizados en China[13],[28] informaron res-

\begin{tabular}{|c|c|c|c|c|c|c|c|c|}
\hline Study or Subgroup & $\begin{array}{l}\text { Aminoqui } \\
\text { Events }\end{array}$ & $\begin{array}{l}\text { olina } \\
\text { Total } \\
\end{array}$ & $\begin{array}{c}\text { Contr } \\
\text { Events }\end{array}$ & rol & Weight & $\begin{array}{c}\text { Risk Ratio } \\
\text { M-H, Random, } 95 \% \mathrm{Cl}\end{array}$ & $\begin{array}{c}\text { Risk Ratio } \\
\text { M-H, Random, } 95 \% \mathrm{Cl} \\
\end{array}$ & \\
\hline $\begin{array}{l}\text { Chen Jun } 2020 \\
\text { Gautret } 2020 \\
\text { Tang } 2020\end{array}$ & $\begin{array}{l}13 \\
14 \\
64\end{array}$ & $\begin{array}{l}15 \\
26 \\
75\end{array}$ & $\begin{array}{r}14 \\
2 \\
61\end{array}$ & $\begin{array}{l}15 \\
16 \\
75\end{array}$ & $\begin{array}{r}43.6 \mathrm{X} \\
5.7 \mathrm{X} \\
50.7 \mathrm{X}\end{array}$ & $\begin{array}{r}0.93[0.73,1.18] \\
4.31[1.12,16.52] \\
1.05[0.91,1.21]\end{array}$ & $t$ & \\
\hline $\begin{array}{l}\text { Total }(95 \% \mathrm{Cl}) \\
\text { Total events }\end{array}$ & 91 & 116 & 77 & 106 & $100.0 \%$ & $1.08[0.77,1.51]$ & & \\
\hline \multicolumn{9}{|c|}{ 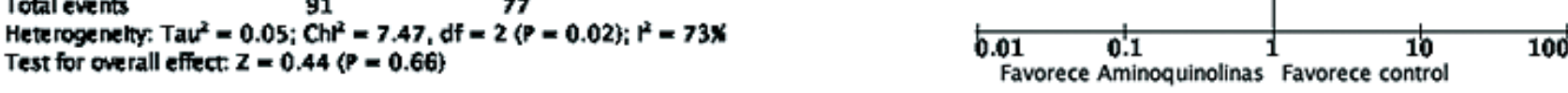 } \\
\hline
\end{tabular}

Figura 3. Aminoquinolinas y probabilidad de negativización del hisopado. 


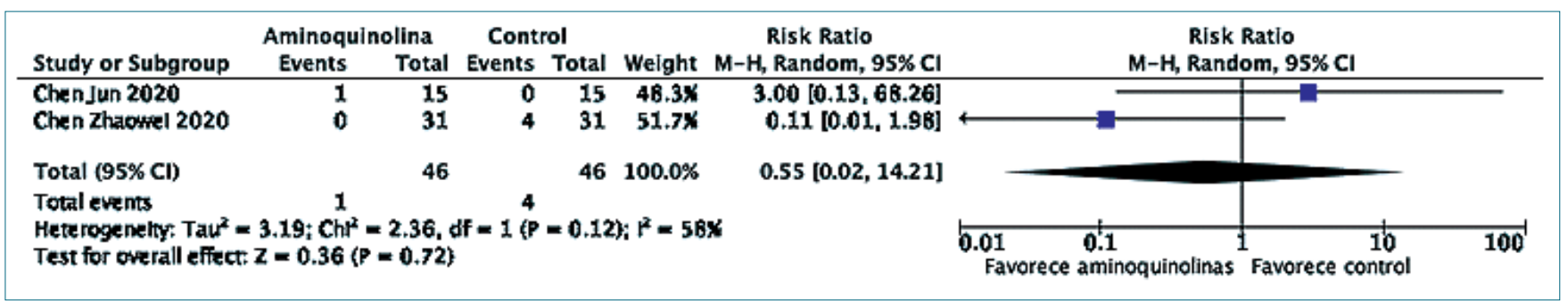

Figura 4. Aminoquinolinas y progresión de enfermedad clínica.

\begin{tabular}{|c|c|c|c|c|c|c|c|c|}
\hline Study or Subgroup & $\begin{array}{l}\text { Aminoqui } \\
\text { Events }\end{array}$ & $\begin{array}{l}\text { olina } \\
\text { Total }\end{array}$ & $\begin{array}{c}\text { Contr } \\
\text { Events }\end{array}$ & rol & Weight & $\begin{array}{l}\text { Risk Ratio } \\
\mathrm{M}-\mathrm{H} \text {, Random, } 95 \% \mathrm{Cl}\end{array}$ & $\begin{array}{l}\text { Risk Ratio } \\
\mathrm{M}-\mathrm{H} \text {, Random, } 95 \% \mathrm{Cl}\end{array}$ & \\
\hline $\begin{array}{l}\text { Chen Jun } 2020 \\
\text { Chen Zhaowel } 2020\end{array}$ & $\begin{array}{l}5 \\
2\end{array}$ & $\begin{array}{l}15 \\
31\end{array}$ & $\begin{array}{l}7 \\
9\end{array}$ & $\begin{array}{l}15 \\
31\end{array}$ & $\begin{array}{l}61.2 \% \\
38.8 \%\end{array}$ & $\begin{array}{l}0.71[0.29,1.75] \\
0.22[0.05,0.95]\end{array}$ & $\longrightarrow$ & \\
\hline Total $(95 \% \mathrm{Cl})$ & & 46 & & 46 & $100.0 \%$ & $0.45[0.14,1.46]$ & & \\
\hline \multicolumn{7}{|c|}{$\begin{array}{l}\text { Total events } \\
\text { Heterogene thy: Tau }=0.37 ; C h r^{2}=1.99, d f=16 \\
\text { Test for overall effect: } Z=1.32\langle P=0.19)\end{array}$} & $\begin{array}{cccc}0.01 & 0.1 & 1 & 10 \\
\text { Favorece } & \text { Aminoquinolinas } & 1 \\
\text { Favorece Control }\end{array}$ & $\overrightarrow{100}$ \\
\hline
\end{tabular}

Figura 5. Aminoquinolinas y probabilidad de progresión radiológica.

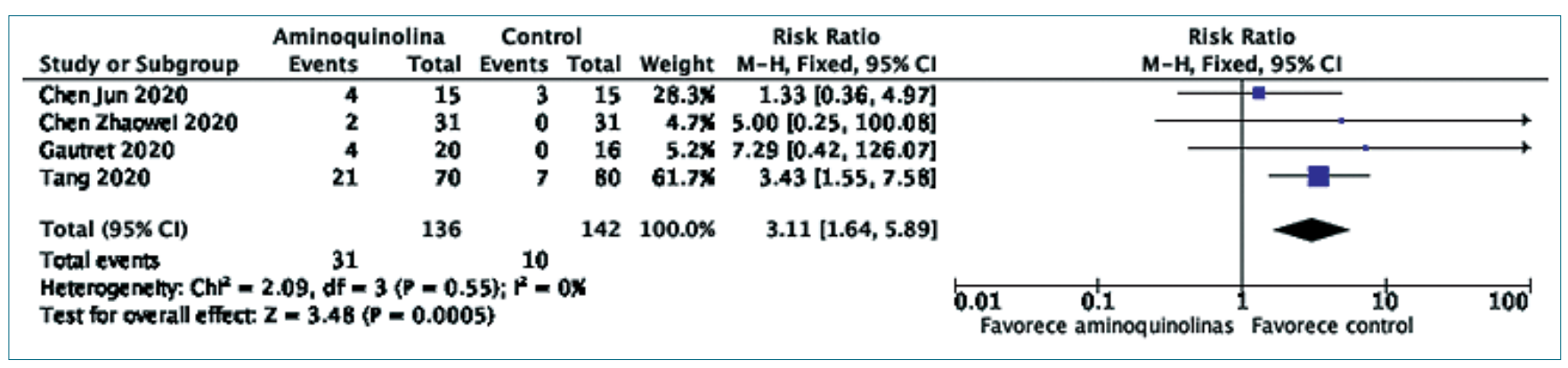

Figura 6. Aminoquinolinas e incidencia de reacciones adversas.

pecto a la probabilidad de progresión radiológica de la enfermedad. Ambos estudios realizaron tomografía computarizada de tórax de control dentro de la primera semana del estudio (tercer y sexto día desde el ingreso) para evaluar la existencia de progresión radiológica. En ninguno de los trabajos se informó la existencia de enmascaramiento de los radiólogos a cargo de la interpretación de las imágenes, por lo que se estimó un alto riesgo de sesgo para la evaluación de este desenlace. Al realizar un metaanálisis de estos estudios, se apreció una reducción en la probabilidad de progresión radiológica (RR: 0,45, IC95\% 0,14 - 1,46 p =0,03) que alcanzó significancia estadística. Al igual que en las comparaciones anteriores, se observó importante heterogeneidad estadística entre resultados ( $Q$ de Cochrane $\left.p=0,16, I^{2}=50 \%\right)$. Se muestra el Forest Plot del metaanálisis en la Figura 5.

\section{Reacciones adversas}

Cuatro estudios agrupando a 278 pacientes reportaron la incidencia de reacciones adversas durante el seguimiento[13],[28],[29],[32]. La mayoría de las reacciones adversas reportadas fueron leves, incluyendo diarrea, náuseas, cefalea, elevación transitoria de transaminasas y reacciones exantemáticas. En un estudio conducido en Marsella, Gautre[t29] repor- tó que uno de los pacientes falleció dentro del grupo de hidroxicloroquina, más no se indicó información respecto a este fallecimiento y no fue considerado como un evento adverso atribuible al medicamento. Al sintetizar los resultados en un metaanálisis, se apreció que el uso de hidroxicloroquina incrementó significativamente la incidencia de reacciones adversas (RR 3,11, IC95\% 1,64-5,89, p < 0,001). En esta oportunidad no se apreció heterogeneidad estadística entre los resultados de los estudios incluidos ( $Q$ de Cochrane $p=0,55,1^{2}=0 \%$ ), permitiendo la utilización de un modelo de eventos fijos para el metaanálisis. Se muestra el Forest Plot de la comparación en la Figura 6.

\section{Cloroquina}

El único ensayo clínico detectado por la búsqueda bibliográfica que evaluó el rol de la cloroquina en el manejo de casos severos de COVID-19 fue el de Silva y colaboradores[30]. Este ensayo clínico aleatorizado evaluó a la cloroquina en dosis de 600 mg c/12 h en comparación al mismo fármaco, pero en dosis menor (450 mg cada 12 h en día 0, luego 450 mg al día hasta día 4 y placebo hasta completar 10 días). Cabe además destacar que este ensayo fue el único conducido entre pacientes con una enfermedad COVID-19 severa y que todos estaban 
recibiendo Azitromicina concomitante a la intervención del ensayo.

Este estudio fue diseñado para incluir 440 pacientes en su protocolo original, más tuvo que ser interrumpido precozmente derivado de un incremento en la mortalidad entre los pacientes asignados a recibir el esquema de alta dosis de cloroquina antes citado. Los pacientes que recibieron el esquema de alta dosis presentaron una letalidad del 39\% (16 de 41), comparado con $15 \%$ (6 de 40) de los asignados al esquema de baja dosis. Este incremento en la mortalidad alcanzó significancia estadística (OR 3,6, IC95\% 1,2 - 10,6). También se apreció una mayor proporción de personas con una prolongación del intervalo QTc sobre 500 milisegundos entre los pacientes asignados al grupo de alta dosis (7 de 37 personas, 18,9\%) en comparación al grupo de control (4 de 36 personas, 11,1\%).

\section{Discusión}

Existe amplia experiencia en el uso de aminoquinolinas como fármacos antiinflamatorios para una gran variedad de condiciones clínicas. Sus efectos en términos de inhibir la replicación viral en modelos in vitro tampoco son nuevos. Varios reportes han mostrado que, in vitro, tanto cloroquina como hidroxicloroquina poseen actividad inhibitoria contra virus influenza A y B, virus de Epstein-Barr, dengue, virus de la inmunodeficiencia humana, entre otros[19],[33]-[35]. No obstante, estos efectos no se han traducido en beneficios para pacientes in vivo en ninguno de los casos anteriores.

Esta revisión sistemática de la literatura buscó evaluar tanto a cloroquina como su metabolito menos tóxico hidroxicloroquina entre pacientes con COVID-19. Ambos fármacos han sido ampliamente recomendados por varios presidentes de países afectados por la pandemia de SARS-CoV-2, incluyendo Estados Unidos y Brasil. Nuestra búsqueda bibliográfica detectó un total de 5 ensayos clínicos aleatorizados relativamente pequeños y con alto riesgo de sesgo que, al integrarlos en un metaanálisis, no mostraron beneficios clínicos ni virológicos entre enfermos de COVID-19. Las fuentes de error sistemático más comunes tuvieron que ver con la falta de enmascaramiento y dificultades en el proceso de aleatorización. Estas formas de error podrían incluso inducir optimismo en la estimación de la eficacia terapéutica de las aminoquinolinas, lo que también debe ser considerado al momento de evaluar estos fármacos para el tratamiento de pacientes individuales.

Nuestros resultados son congruentes con un estudio de cohorte y un ensayo clínico orientado a prevención primaria publicados mientras se realizaba esta revisión. En el primero de ellos[22], Geleris y colaboradores evaluaron desenlaces clínicos en una muestra consecutiva de 1.446 pacientes, de los cuales el 58,9\% recibió hidroxicloroquina. Para facilitar las comparaciones, los investigadores condujeron un análisis de pareo por puntaje de propensión, limitando los efectos de variables de confusión en sus resultados. No se apreciaron diferencias entre los grupos tratados con y sin hidroxicloroquina en términos de desarrollar una falla respiratoria que requiriera intubación o mortalidad (HR 1,04, IC95\% 0,82-1,32). Este resultado se mantuvo robusto en múltiples análisis de sensibilidad. En el segundo trabajo, Boulware[36] y colaboradores evaluaron el uso de hidroxicloroquina entre pacientes en riesgo de contraer in- fección a SARS-CoV-2. El estudio no demostró una reducción en la incidencia de COVID-19 entre los participantes tratados, más sí detectó un incremento de 2,5 veces en la incidencia de reacciones adversas, la que alcanzó significancia estadística. El retiro de participantes del estudio fue más común entre pacientes que recibieron hidroxicloroquina, fundamentalmente debido a la aparición de estos últimos eventos.

El único desenlace en el que se apreció un beneficio estadísticamente significativo fue la probabilidad de progresión radiológica. En base a dos estudios en alto riesgo de sesgo, hidroxicloroquina cuantificó una reducción del $55 \%$ en la probabilidad de progresión radiológica (RR: 0,45, IC95\% 0,14 - 1,46 p = $0,03)$. No obstante, este resultado debe ser interpretado con especial cautela. La falta de enmascaramiento y cuantificación de variabilidad interobservador entre los médicos encargados de interpretar las imágenes son fuentes de sesgo específicas que afectan la fiabilidad de estos estimadores en forma relevante. Por otro lado, debe también considerarse que aún cuando existiera una mejoría en términos de progresión radiológica, ésta estaría disociada de otros desenlaces asociados a la misma, como aclaramiento virológico, deterioro clínico y mortalidad.

A diferencia de la revisión de Sarma y colaboradores[37], el metaanálisis respecto a la incidencia de reacciones adversas detectó un incremento significativo en el riesgo de estos eventos entre pacientes tratados (RR 3,11, IC95\% 1,64-5,89, p < $0,001)$. La razón está en la inclusión adicional de un ensayo clínico aleatorizado de tamaño considerable, el que permitió alcanzar la potencia estadística suficiente para detectar este contraste. Si bien la mayoría de las reacciones adversas reportadas fueron menores, debe considerarse que la mayoría de estos estudios sólo incluyeron pacientes con formas leves de enfermedad, susceptibles de ser tratados en forma ambulatoria. Sólo el trabajo de Silva fue realizado expresamente entre pacientes críticos[30], mientras que un segundo[32] consideró la inclusión post-hoc de pacientes de estas características al término del trabajo, por lo que sólo ingresaron dos enfermos en esta condición. Considerando la carga de morbilidad y disfunciones orgánicas que este subgrupo de pacientes suele presentar[4],[38], es posible que el riesgo de estos eventos se vea incluso incrementado entre estos pacientes.

Otra consideración relevante está en que la mayoría de los estudios incluidos utilizaron una aminoquinolina en combinación con otros fármacos, incluyendo corticoides, interferón y antimicrobianos, entre los que se incluye a la azitromicina. Este último fármaco es de especial interés considerando sus efectos en el prolongar el intervalo QT, pudiendo facilitar la aparición de arritmias ventriculares malignas. Sólo un estudio que trabajó con cloroquina en alta dosis reportó una mayor incidencia de QT prolongado y mortalidad[30], por lo que tuvo que ser suspendido en forma prematura.

\section{Limitantes del estudio}

Esta revisión sistemática tiene algunas limitaciones. Si bien se realizó una búsqueda de la literatura sensible en múltiples repositorios, lo que llevó a detectar e incluir trabajos no detectados en revisiones anteriores, debe considerarse que la mayoría de los trabajos incurrieron en un alto riesgo de sesgo. Esto restringe importantemente la calidad de las conclusiones que pueden tomarse a partir del metaanálisis, puesto que podrían 
inducir optimismo en los estimadores obtenidos. Por otro lado, la falta de inclusión de pacientes hospitalizados o críticamente enfermos restringe la aplicación de esta evidencia a estas subpoblaciones, con la consideración de que podría existir un mayor riesgo de reacciones adversas entre estos enfermos.

El relativamente pequeño número de participantes restringió además la evaluación de fuentes de heterogeneidad de los trabajos incluidos, las que son siempre importantes de explorar para orientar la investigación futura en esta área.

\section{Conclusión}

La evidencia disponible no apoya la utilización rutinaria de aminoquinolinas en el tratamiento de pacientes con COVID-19. Los beneficios teóricos derivados de modelos in-vitro no se traducen en desenlaces clínicos importantes. Existió un incremento significativo en la frecuencia de reacciones adversas, aún en población con formas leves de la enfermedad, las que pueden ser más comunes entre pacientes gravemente enfermos.

\section{Referencias}

1. Zhu N, Zhang D, Wang W, Li X, Yang B, Song J, et al.; China Novel Coronavirus Investigating and Research Team. A novel coronavirus from patients with pneumonia in China, 2019. N Engl J Med. 2020 Feb;382(8):727-33. https://doi.org/10.1056/ NEJMoa2001017 PMID:31978945

2. Guan WJ, Ni ZY, Hu Y, Liang WH, Ou CQ, He JX, et al.; China Medical Treatment Expert Group for Covid-19. Clinical Characteristics of Coronavirus Disease 2019 in China. N Engl J Med. 2020 Apr;382(18):1708-20. https://doi.org/10.1056/NEJMoa2002032 PMID:32109013

3. $X u X W, W u X X$, Jiang $X G, X u K J$, Ying $L J, M a C L$, et al. Clinical findings in a group of patients infected with the 2019 novel coronavirus (SARS-Cov-2) outside of Wuhan, China: retrospective case series. BMJ. 2020 Feb;368(January):m606. https://doi. org/10.1136/bmj.m606 PMID:32075786

4. Yang $X, Y u Y, X u$ J, Shu H, Xia J, Liu H, et al. Clinical course and outcomes of critically ill patients with SARS-CoV-2 pneumonia in Wuhan, China: a single-centered, retrospective, observational study. Lancet Respir Med. 2020 May;8(5):475-81. https://doi. org/10.1016/S2213-2600(20)30079-5 PMID:32105632

5. Berlin DA, Gulick RM, Martinez FJ. Severe Covid-19.. N Engl J Med. 2020 May 15;NEJMcp2009575.

6. Zhou F, Yu T, Du R, Fan G, Liu Y, Liu Z, et al. Clinical course and risk factors for mortality of adult inpatients with COVID-19 in Wuhan, China: a retrospective cohort study. Lancet. 2020 Mar;395(10229):1054-62. https://doi.org/10.1016/S01406736(20)30566-3 PMID:32171076

7. Lippi G, Plebani M. Laboratory abnormalities in patients with COVID-2019 infection. Clin Chem Lab Med. 2020 Jun;58(7):1131-4. https://doi.org/10.1515/cclm-2020-0198 PMID:32119647

8. Wang D, Hu B, Hu C, Zhu F, Liu X, Zhang J, et al. Clinical Characteristics of 138 Hospitalized Patients With 2019 Novel Coronavirus-Infected Pneumonia in Wuhan, China. JAMA. 2020 Mar;323(11):1061-9. https://doi.org/10.1001/jama.2020.1585 PMID:32031570

9. Sanders JM, Monogue ML, Jodlowski TZ, Cutrell JB. Pharma- cologic Treatments for Coronavirus Disease 2019 (COVID-19): A Review. JAMA. 2020 May;323(18):1824-36. https://doi. org/10.1001/jama.2020.6019 PMID:32282022

10. Xu X, Han M, Li T, Sun W, Wang D, Fu B, et al. Effective Treatment of Severe COVID-19 Patients with Tocilizumab. chinaXiv. 2020;1-12. https://doi.org/10.1073/pnas.2005615117.

11. Zhang $L$, Liu $Y$. Potential interventions for novel coronavirus in China: A systematic review. J Med Virol. 2020 May;92(5):479-90. https://doi.org/10.1002/jmv.25707 PMID:32052466

12. Liu J, Cao R, Xu M, Wang X, Zhang H, Hu H, et al. Hydroxychloroquine, a less toxic derivative of chloroquine, is effective in inhibiting SARS-CoV-2 infection in vitro. Cell Discov. 2020 Mar;6(1):16. https://doi.org/10.1038/s41421-020-0156-0 PMID:32194981

13. Chen Z, Hu J, Zhang Z, Jiang S, Han S, Yan D, et al. Efficacy of hydroxychloroquine in patients with COVID-19: results of a randomized clinical trial. medRxiv. 2020;7:2020.03.22.20040758. https://doi.org/10.1101/2020.03.22.20040758.

14. Cortegiani A, Ingoglia G, Ippolito M, Giarratano A, Einav S. A systematic review on the efficacy and safety of chloroquine for the treatment of COVID-19. J Crit Care. 2020 Jun;57:279-83. https://doi.org/10.1016/j.jcrc.2020.03.005 PMID:32173110

15. Yao $X$, Ye F, Zhang M, Cui C, Huang B, Niu P, et al. In Vitro Antiviral Activity and Projection of Optimized Dosing Design of Hydroxychloroquine for the Treatment of Severe Acute Respiratory Syndrome Coronavirus 2 (SARS-CoV-2). Clin Infect Dis. 2020;(Xx):1-8.

16. Wang $M$, Cao R, Zhang L, Yang $X$, Liu J, Xu M, et al. Remdesivir and chloroquine effectively inhibit the recently emerged novel coronavirus (2019-nCoV) in vitro. Cell Res. 2020 Mar;30(3):269-71. https://doi.org/10.1038/s41422-020-0282-0 PMID:32020029

17. Keyaerts E, Vijgen L, Maes P, Neyts J, Van Ranst M. In vitro inhibition of severe acute respiratory syndrome coronavirus by chloroquine. Biochem Biophys Res Commun. 2004 Oct;323(1):264-8. https://doi.org/10.1016/j.bbrc.2004.08.085 PMID:15351731

18. Shah S, Das S, Jain A, Misra DP, Negi VS. A systematic review of the prophylactic role of chloroquine and hydroxychloroquine in coronavirus disease-19 (COVID-19). Int J Rheum Dis. 2020 May;23(5):613-9. https://doi.org/10.1111/1756-185X.13842 PMID:32281213

19. Ferner RE, Aronson JK. Chloroquine and hydroxychloroquine in covid-19. BMJ. 2020 Apr;369(April):m1432. https://doi. org/10.1136/bmj.m1432 PMID:32269046

20. Vincent MJ, Bergeron E, Benjannet S, Erickson BR, Rollin PE, Ksiazek TG, et al. Chloroquine is a potent inhibitor of SARS coronavirus infection and spread. Virol J. 2005 Aug;2(1):69. https:// doi.org/10.1186/1743-422X-2-69 PMID:16115318

21. Meo SA, Klonoff DC, Akram J. Efficacy of chloroquine and hydroxychloroquine in the treatment of COVID-19. Eur Rev Med Pharmacol Sci. 2020 Apr;24(8):4539-47. PMID:32373993

22. Geleris J, Sun Y, Platt J, Zucker J, Baldwin M, Hripcsak G, et al. Observational Study of Hydroxychloroquine in Hospitalized Patients with Covid-19. N Engl J Med. 2020 Jun;382(25):2411-8. https:// doi.org/10.1056/NEJMoa2012410 PMID:32379955

23. Mehra MR, Desai SS, Ruschitzka F, Patel AN. Hydroxychloroquine or chloroquine with or without a macrolide for treatment of COVID-19: a multinational registry analysis. Lancet. 2020;6736(20):1-10.

24. Liberati A, Altman DG, Tetzlaff J, Mulrow C, Gøtzsche PC, loannidis JP, et al. The PRISMA statement for reporting sys- 
tematic reviews and meta-analyses of studies that evaluate health care interventions: explanation and elaboration. PLoS Med. 2009 Jul;6(7):e1000100. https://doi.org/10.1371/journal. pmed.1000100 PMID:19621070

25. Higgins J, Thomas J, Chandler J, Cumpston M, Li T, Page M, et al. Cochrane Handbook for Systematic Reviews of Interventions [Internet]. Higgins JPT, Thomas J, Chandler J, Cumpston M, Li T, Page MJ, et al., editors. Cochrane Handbook for Systematic Reviews of Interventions. Wiley; 2019.

26. Xu H, Platt RW, Luo ZC, Wei S, Fraser WD. Exploring heterogeneity in meta-analyses: needs, resources and challenges. Paediatr Perinat Epidemiol. 2008 Jan;22(s1 Suppl 1):18-28. https://doi. org/10.1111/j.1365-3016.2007.00908.x PMID:18237348

27. Khoshdel A, Attia J, Carney SL. Basic concepts in metaanalysis: A primer for clinicians [Internet]. Int J Clin Pract. 2006 Oct;60(10):1287-94. https://doi.org/10.1111/j.17421241.2006.01078.x PMID:16981972

28. Chen J, Liu D, Liu L, Liu P, Xu Q, Xia L, et al. [A pilot study of hydroxychloroquine in treatment of patients with moderate COVID-19]. Zhejiang Da Xue Xue Bao Yi Xue Ban. 2020 May;49(2):215-9. PMID:32391667

29. Gautret P, Lagier JC, Parola P, Hoang VT, Meddeb L, Mailhe $M$, et al. Hydroxychloroquine and azithromycin as a treatment of COVID-19: results of an open-label non-randomized clinical trial. Int J Antimicrob Agents. 2020 Jul;56(1):105949. https://doi. org/10.1016/j.ijantimicag.2020.105949 PMID:32205204

30. Borba MGS, Val FFA, Sampaio VS, Alexandre MAA, Melo GC, Brito $M$, et al. Effect of High vs Low Doses of Chloroquine Diphosphate as Adjunctive Therapy for Patients Hospitalized With Severe Acute Respiratory Syndrome Coronavirus 2 (SARS-CoV-2) Infection. JAMA Netw Open. 2020;3(4.23):e208857.

31. Ferro F, Elefante E, Baldini C, Bartoloni E, Puxeddu I, Talarico R, et al. COVID-19: the new challenge for rheumatologists. Clin Exp Rheumatol. 2020 Mar-Apr;38(2):175-80. PMID:32207680

32. Tang W, Cao Z, Han M, Wang Z, Chen J, Sun W, et al. Hydroxychloroquine in patients mainly with mild to moderate $\mathrm{CO}$ VID-19: an open-label, randomized, controlled trial. medRxiv. 2020 Jan 1;2020.04.10.20060558. https://doi.org/10.1101/2020 04.10.20060558.

33. Vigerust DJ, McCullers JA. Chloroquine is effective against influenza A virus in vitro but not in vivo. Influenza Other Respir Viruses. 2007 Sep-Nov;1(5-6):189-92. https://doi.org/10.1111/ j.1750-2659.2007.00027.x PMID:19453426

34. Wang LF, Lin YS, Huang NC, Yu CY, Tsai WL, Chen JJ, et al. Hydroxychloroquine-inhibited dengue virus is associated with host defense machinery. J Interferon Cytokine Res. 2015 Mar;35(3):143-56. https://doi.org/10.1089/jir.2014.0038 PMID:25321315

35. Savarino A, Boelaert JR, Cassone A, Majori G, Cauda R. Effects of chloroquine on viral infections: an old drug against today's diseases? Lancet Infect Dis. 2003 Nov;3(11):722-7. https://doi. org/10.1016/S1473-3099(03)00806-5 PMID:14592603

36. Boulware DR, Pullen MF, Bangdiwala AS, Pastick KA, Lofgren SM, Okafor EC, et al. A Randomized Trial of Hydroxychloroquine as Postexposure Prophylaxis for Covid-19. N Engl J Med. 2020 Aug;383(6):517-25. https://doi.org/10.1056/NEJMoa2016638 PMID:32492293

37. Sarma $P$, Kaur $H$, Kumar $H$, Mahendru D, Avti $P$, Bhattacharyya A, et al. Virological and clinical cure in COVID-19 patients treated with hydroxychloroquine: A systematic review and metaanalysis. J Med Virol [Internet]. 2020 May 3;jmv.25898.

38. Murthy S, Gomersall CD, Fowler RA. Care for Critically III Patients With COVID-19. JAMA. 2020 Apr;323(15):1499-500. https://doi. org/10.1001/jama.2020.3633 PMID:32159735 